\title{
Middle East respiratory syndrome coronavirus (MERS-Cov) screening of exposed healthcare workers in a tertiary care hospital in Saudi Arabia
}

\author{
K Alameer ${ }^{* *}$, B Abukhzam², W Khan', A El-Saed ${ }^{1}$, H Balkhy \\ From 3rd International Conference on Prevention and Infection Control (ICPIC 2015) \\ Geneva, Switzerland. 16-19 June 2015
}

\begin{abstract}
Introduction
The possibility of healthcare exposure to Middle East Respiratory Syndrome Coronavirus (MERS-Cov) has been early described. Almost one-third of the confirmed MERS-Cov cases in Saudi Arabia were among healthcare workers (HCWs).
\end{abstract}

\section{Objectives}

To describe three-season experience of MERS-Cov exposure and outcome among HCWs in a tertiary care hospital in Saudi Arabia.

\section{Methods}

Prospective surveillance was conducted in King Abdulaziz Medical City in Riyadh, Saudi Arabia for unprotected exposed HCWs, with every newly PCR-confirmed MERS-Cov case, between June 2013 and March 2015. HCWs exposed to confirmed MERS-Cov patients were examined for the presence of symptoms and nasopharyngeal (and rarely other) swab was obtained for MERS-Cov. Exposure was defined as caring of or being in close proximity (within 2 meters) of a confirmed patient, without proper personal protective equipment (PPE).

\section{Results}

During the duration covered, a total 32 patients with PCR-confirmed MERS-Cov were associated with exposure of $1361 \mathrm{HCWs}$. Only 328 (24.1\%) of the exposed HCWs had symptoms suggestive of respiratory infection at the time of screening. MERS-Cov was confirmed in only $14(1.03 \%)$ HCWs. MERS-Cov confirmation was roughly similar among symptomatic $(3 / 328,0.91 \%)$ and asymptomatic (11/1033, 1.06\%) HCWs. Only 2 (14.3\%) of the 14 confirmed $\mathrm{HCWs}$ required hospitalization. While the mortality among the confirmed patients was very high $(21 / 32,65.6 \%)$, none of the 14 confirmed healthcare workers died.

\section{Conclusion}

We are reporting high potential of healthcare exposure to MERS-Cov in the healthcare setting but a very low transmission rate. Proper compliance with PPEs is essential to further reduce unprotected exposure of the HCWS. In essence contact tracing and testing for all unprotected exposed HCWs to MERS CoV irrespective of symptoms remain critical measures to prevent or reduce the impact of MERS-Cov hospital outbreak till further understanding of MERS-Cov behavior is delineated.

\section{Disclosure of interest}

None declared.

\section{Authors' details}

'Infection Prevention and Control, Riyadh, Saudi Arabia. ${ }^{2}$ King Abdulaziz Medical City, Riyadh, Saudi Arabia.

Published: 16 June 2015

doi:10.1186/2047-2994-4-S1-057

Cite this article as: Alameer et al: Middle East respiratory syndrome coronavirus (MERS-Cov) screening of exposed healthcare workers in a tertiary care hospital in Saudi Arabia. Antimicrobial Resistance and Infection Control 2015 4(Suppl 1):057.

IIfection Prevention and Control, Riyadh, Saudi Arabia

Full list of author information is available at the end of the article 